\title{
Erfassung der Tagesschläfrigkeit mittels Epworth- Schläfrigkeits-Skala und einem 3-Item-Fragebogen bei obstruktiver Schlafapnoe
}

\author{
Detection of Daytime Sleepiness Using Epworth Sleepiness Scale and \\ a 3-Item Questionnaire in Obstructive Sleep Apnea
}

Autoren

Institute
K. H. Rühle ${ }^{1,3}$, O. Kuhtin ${ }^{2}$, U. Domanski ${ }^{1}$, K. J. Franke ${ }^{1,3}$, G. Nilius ${ }^{1,3}$

${ }^{1}$ Klinik für Pneumologie, HELIOS-Klinik Ambrock Hagen

${ }^{2}$ HELIOS Klinik Krefeld, Thoraxchirurgie

${ }^{3}$ Universität Witten-Herdecke eingereicht $\quad 30.11 .2011$ akzeptiert nach Revision 21. 12. 2011

\section{Bibliografie}

Dol http://dx.doi.org/ 10.1055/s-0031-1291634 Online-Publikation: 27.1.2012 Pneumologie 2012; 66: 184-187 (c) Georg Thieme Verlag KG Stuttgart · New York ISSN 0934-8387

Korrespondenzadresse Prof. Dr. med. Karl-Heinz Rühle HELIOS-Klinik Ambrock Klinik für Pneumologie Ambrockerweg 60 58091 Hagen Klinik-Ambrock.Pneumo@ t-online.de

\section{Zusammenfassung \\ V}

Einleitung: In der Epworth-Schläfrigkeits-Skala (ESS) wird nach der Wahrscheinlichkeit des Einschlafens in 8 spezifischen Situationen gefragt. Pathologische Schläfrigkeit (TS) von Patienten mit obstruktiver Schlafapnoe (OSA) wird am häufigsten bei einem ESS-Score $\geq 11$ diagnostiziert (TSESS). In einer epidemiologischen Studie zur Prävalenz des Schlafapnoe-Syndroms (OSAS) wurden zur Charakterisierung pathologischer Tagesschläfrigkeit (TS-Young) lediglich 3 Fragen mit JaNein-Antworten verwandt: Aufgrund der unterschiedlichen Fragenkonstruktion der ESS und des Young-Fragebogens fragten wir, ob im Vergleich zur ESS eine größere Anzahl von Patienten mit OSA und TS mit der Kombination beider Fragebögen identifiziert wird. Zusätzlich überprüften wir die Schläfrigkeitsfragebögen (FB) an mittels objektivem Vigilanztest definierten Gruppen von OSA-Patienten.

Methodik: Mittels PSG wurden 328 OSA-Patienten mit einem AHI $\geq 5$ identifiziert. Die Vigilanz wurde mit dem Computerprogramm CARDA untersucht. Ergebnisse: Mit beiden FB fand sich mit $48 \%$ derselbe Prozentsatz von Patienten mit AHI $>5$ mit pathologischer Schläfrigkeit. Durch Kombination beider FB (TS-Komb) erhöhte sich die Anzahl der Patienten von 158 (48,2\%) auf 195 (59,5\%) signifikant. Die Kombination ergab damit eine signifikant höhere Trefferquote mit zusätzlich 37 (11,3\%) Patienten im Vergleich zur TS-ESS allein. Kombiniert man bei Patienten mit unterdurchschnittlicher Fehlerzahl $(n=192)$ im Vigilanztest beide FB, resultiert eine Zunahme von TS von $23,2 \%$ auf $31,4 \%$ dieser Fälle $p<0,001$.

Schlussfolgerungen: Als Einzeltest können beide Fragebögen mit gleicher Trefferquote für Tagesschläfrigkeit verwendet werden. Durch die Kombination beider Fragebögen findet man im Vergleich zur ESS allein besonders bei Patienten ohne Einschränkung der Vigilanz einen höheren Prozentsatz von Tagesschläfrigkeit.

\section{Abstract \\ $\nabla$}

Introduction: The Epworth Sleepiness Scale (ESS) describes the likelihood of falling asleep in 8 specific situations. Pathological sleepiness (TS) of patients with obstructive sleep apnea (OSA) is most often diagnosed with an ESS score $\geq 11$ (TSESS). In an epidemiological study on the prevalence of sleep apnea syndrome (OSAS), only three questions with yes-no answers were used for the characterization of pathological daytime sleepiness (TS-Young): Due to the different construction of the ESS and the Young's questionnaire, we asked whether with the combination of the two questionnaires a larger number of patients with OSA and TS compared to the ESS can be identified. In addition, we examined the sleepiness questionnaires (FB) using objective vigilance in defined groups of OSA patients.

Methods: Using PSG 328 OSA patients with an $A H I \geq 5$ were identified. Vigilance was examined using the computer program CARDA.

Results: With both FB the same percentage of patients with $\mathrm{AHI}>5$ with pathological sleepiness was found $(48 \%)$. By combining both FB (TScomb), the number of patients increased from $158(48.2 \%)$ to 195 (59.5\%) significantly. The combination showed a significantly higher percentage with additional $37(11.3 \%)$ patients in comparison to the TS-ESS alone. Combining both $\mathrm{FB}$, an increase of TS resulted in patients with below-average number of errors in the vigilance test $(\mathrm{n}=192)$ from $23.2 \%$ to $31.4 \%$ of these cases $(\mathrm{p}<0.001)$.

Conclusions: As a single test both questionnaires can be used to detect daytime sleepiness with equal incidence. Combining both questionnaires a higher percentage of daytime sleepiness can be found in comparison to the ESS-questionnaire alone especially in patients without disturbance of vigilance. 


\section{Einleitung}

\section{$\nabla$}

Eines der wichtigsten Symptome bei schlafbezogenen Atmungsstörungen insbesondere beim obstruktiven Schlafapnoe-Syndrom (OSAS) stellt die Tagesschläfrigkeit (TS) dar. Ihre Wertigkeit wird u. a. dadurch offensichtlich, dass viele Verkehrsunfälle durch Schläfrigkeit am Steuer ausgelöst werden [1]. Die berichtete, subjektive Schläfrigkeit der Patienten kann mit verschiedenen Fragebögen charakterisiert werden. Am häufigsten wird die EpworthSleepiness-Scale (ESS) verwendet, welche die Schlafneigung bei acht spezifischen Situationen mit unterschiedlichem Wachdruck beschreibt [2-6]. Pathologische TS besteht bei einem ESS-Score $\geq 11$ (TS-ESS) [7]. Eine Studie im deutschen Sprachraum bestätigte diesen Grenzwert [8].

In einer früheren epidemiologischen Studie zur Prävalenz des Schlafapnoe-Syndroms wurden zur Charakterisierung der Tagesschläfrigkeit die 3 folgenden Beschwerden verwandt [9]: Mehr als an 2 Tagen in der Woche auftretende

- Tagesschläfrigkeit,

- unerfrischtes Aufwachen,

- unkontrollierbare Schläfrigkeit, die im Tagesablauf stört.

Zur Definition von Hypersomnolenz (TS) wurde die positive Beantwortung aller 3 Fragen verwandt (TS-Young). Bei einem AHI $>5 / h$ und TS-Young wurde bei Männern eine Prävalenz von 4\% und bei Frauen von $2 \%$ gefunden.

Da die Fragen der ESS nach Wahrscheinlichkeiten von manchen Patienten nicht valide ausgefüllt werden können, untersuchten wir, ob die Fragenkombination nach Young mit einfacher, dichotomer Skalierung und Beantwortung der Fragen mit Ja oder Nein im Vergleich zur ESS einen höheren Prozentsatz von Patienten mit OSA und TS erfasst. Vergleichende Untersuchungen zur Tagesschläfrigkeit mit der ESS und dem von uns verwendeten Fragebogen mit den Fragen nach T. Young wurden bisher nicht durchgeführt.

In einem ersten Schritt verglichen wir die beiden Tests an einer OSA-Patienten-Gruppe, die wir mittels AHI > 5 definierten.

Allerdings wird bei diesem Vorgehen kein objektives Kriterium zur Charakterisierung des Schweregrades der Schläfrigkeit benutzt.

In einer Untersuchung von Adams [10] konnte gezeigt werden, dass eine negative Assoziation zwischen Schläfrigkeit und Vigilanz besteht.

Zum Nachweis einer verminderten Vigilanz stand uns ein Vigilanztest (Carda) [11], ähnlich dem häufig eingesetzten Vigilanztest (Steer Clear), zur Verfügung [12].

Damit war es möglich, in einem zweiten Schritt die Schläfrigkeitsfragebögen an verschiedenen Gruppen von OSA-Patienten, definiert mit einem objektiven Testverfahren, zu vergleichen.

\section{Methodik}

$\nabla$

Ausgewertet wurden retrospektiv die Daten von 350 konsekutiven Patienten, die im ersten Halbjahr 2009 stationär im Schlaflabor der Klinik zur Diagnostik bei Verdacht auf obstruktive Schlafapnoe aufgenommen wurden. Am Tag der Aufnahme beantwortete der Patient sowohl den ESS-Fragebogen als auch einen Fragebogen mit den 3 Fragen nach Young. Als Tagesschläfrigkeit wurde gewertet, wenn der ESS-Score $\geq 11$ betrug und dann als TS-ESS bezeichnet. Weiterhin wurde als Tagesschläfrigkeit gewertet, wenn alle 3 Fragen nach Young positiv beantwortet wurden, und dann als TS-Young bezeichnet. Die diagnostische
Polysomnografie erfolgte in der ersten Nacht nach Aufnahme. Am 2. Tag nach Aufnahme wurde jeweils der Vigilanztest durchgeführt. Die Auswertung der PSG erfolgte nach den Kriterien von Rechtschaffen und Kales (R\&K).

Bei dem Vigilanztest Carda handelt es sich um ein Turbo-PascalProgramm, bei dem auf einem Bildschirm mit dunklem Hintergrund eine Straße mit einem Mittel- und Seitenstreifen dargestellt wird. Intermittierend und zufällig werden auf der Straße für die Dauer von 20 ms mit einer Häufigkeit von 100 Ereignissen pro $10 \mathrm{~min}$ rechteckige Hindernisse gezeigt. Der Patient wurde gebeten, auf ein Hindernis mit dem Tippen der Leertaste zu reagieren. Als Fehler wurde gewertet, wenn innerhalb einer Sekunde keine Reaktion erfolgte. Die Testdauer betrug für alle Patienten $30 \mathrm{~min}$. Die prozentuale Fehlerrate wurde aus der Anzahl der Fehler, bezogen auf die Gesamtzahl der präsentierten Hindernisse berechnet. Die mittlere Fehlerzahl bei einer Gruppe von 51 Probanden lag bei 4,7 $\pm 4,3 \%$ [11].

Auswertung: Zur Untersuchung der Frage, ob die Vigilanz bei Kombination beider FB die Quote für Tageschläfrigkeit beeinflusst, bildeten wir anhand des Mittelwertes der Fehler im Vigilanztest 2 Gruppen mit unterdurchschnittlicher ( $\geq 5 \%$ Fehler) bzw. überdurchschnittlicher Vigilanz ( $<5 \%$ Fehler ) bezogen auf die gesamten OSA-Gruppe $(n=328)$. Schließlich untersuchten wir die Kombination beider FB bei einer Gruppe mit eingeschränkter Vigilanz, die wir anhand einer Fehlerrate oberhalb des Mittelwertes plus Standardabweichung (>9\% Fehlerrate) definierten.

Ergebnisse: Bei 22 Fällen mit einem $\mathrm{AHI}<5$ wurde die Diagnose einer OSA ausgeschlossen. Unter den 328 verbleibenden Patienten befanden sich 273 (83,2\%) Männer und 55 (16,8\%) Frauen. Das Alter lag bei 53,5 $\pm 11,6$ Jahren (19 bis 79 Jahren). Das auf die Körpergröße bezogene Gewicht aller Patienten (Body Mass Index, BMI) lag im Mittel bei 32,0 \pm 6,2 (siehe Tab. 1).

Tab. 1 Anthropometrische und polysomnografische Daten der untersuchten Patienten.

\begin{tabular}{|llll|}
\hline & $\begin{array}{l}\text { Gesamtes Patienten- } \\
\text { kollektiv }\end{array}$ & Männer & Frauen \\
\hline Patientenzahl & 328 & $273(83,2 \%)$ & $55(16,8 \%)$ \\
\hline Alter & $53,5 \pm 11,6$ & $53,4 \pm 11,7$ & $54,3 \pm 12,6$ \\
\hline $\begin{array}{l}\text { Größe (cm) } \\
\text { Gewicht (kg) }\end{array}$ & $175,7 \pm 8,7$ & $177,7 \pm 7,9$ & $165,9 \pm 6,6$ \\
\hline $\mathrm{BMI}$ & $32,0 \pm 21,4$ & $100,3 \pm 20,6$ & $91,5 \pm 23,4$ \\
\hline $\mathrm{AHI}$ & $36,0 \pm 25,6$ & $31,7 \pm 5,9$ & $33,1 \pm 7,6$ \\
\hline $\mathrm{SO}_{2}$ min (\%) & $76,9 \pm 10,7$ & $37,8 \pm 25,5$ & $27,3 \pm 24,8$ \\
\hline $\begin{array}{l}\mathrm{SO}_{2} \\
\text { mittl. NREM (\%) }\end{array}$ & $91,9 \pm 3,1$ & $76,9 \pm 10,8$ & $77,3 \pm 10,2$ \\
\hline $\begin{array}{l}\mathrm{SO}_{2} \\
\text { mittl. REM (\%) }\end{array}$ & $87,9 \pm 17,2$ & $87,6 \pm 3,1$ & $92,1 \pm 2,8$ \\
\hline $\begin{array}{l}\text { Vigilanztest } \\
\text { (Fehler \%) }\end{array}$ & $7,3 \pm 9,8$ & $6,7 \pm 9,8$ & $10,2 \pm 9,3$ \\
\hline ESS-Score & $10,4 \pm 5,3$ & $10,4 \pm 5,3$ & $10,5 \pm 5,4$ \\
\hline
\end{tabular}

Zwischen der Zahl der Patienten mit TS-ESS n=158/273 (48,2\%) und TS-Young, $\mathrm{n}=156 / 273$ (47,6\%), besteht im Chi $^{2}$-Test kein signifikanter Unterschied ( $\mathrm{p}=0,909)$ (siehe Tab.2).

Berücksichtigt man von jedem Patienten einen pathologischen ESS-Score und/oder einen pathologischen Young-Score, erhöht sich die Anzahl der Patienten von 158 (48,2\%) auf 195 (59,5\%) signifikant. 
Tab.2 Anzahl und Prozentsatz der Patienten mit und ohne Hypersomnolenz, definiert anhand des Grenzwertes der ESS (TS-ESS) bzw. des YoungFragebogens (TS-Young).

\begin{tabular}{llc|}
$\begin{array}{l}\text { Patientenkollektiv } \\
\text { (n=328) AHI>5 }\end{array}$ & $\begin{array}{l}\text { TS-Young-positiv } \\
\text { (n=156, 47,6\%) }\end{array}$ & $\begin{array}{l}\text { TS-Young-negativ } \\
\text { (n=172, 52,4\%) }\end{array}$ \\
\hline $\begin{array}{l}\text { TS-ESS-positiv } \\
(\mathbf{n}=158,48,2 \%)\end{array}$ & $133(40,5 \%)$ & $39(11,9 \%)$ \\
\hline $\begin{array}{l}\text { TS-ESS-negativ } \\
(\mathbf{n}=170,51,8 \%)\end{array}$ & $37(11,3 \%)$ & $119(36,3 \%)$ \\
\hline
\end{tabular}

Eine Kombination aus den beiden Fragebögen mit TS-ESS und TSYoung zeigt damit eine signifikant höhere Trefferquote mit zusätzlich 37 (11,3\%) Patienten im Vergleich zur TS-ESS allein. Der Unterschied ist auf dem Niveau $\mathrm{p}<0,01$ (McNemar Test) signifikant.

Die Anzahl der Fälle, bei denen das Ergebnis bzgl. Tagesschläfrigkeit übereinstimmt bzw. divergiert, ist aus der $\bullet$ Tab. 2 ersichtlich.

Wir fanden eine Übereinstimmung von TS-ESS und TS-Young in 133 Fällen (40,5\%) und keine TS-ESS und TS-Young bei 119 Fällen (36,3\%).

Eine Divergenz der Ergebnisse sehen wir in 76 (39 und 37) Fällen (23,2\%).

Der Prozentsatz an Patienten mit Schläfrigkeit bei unterdurchschnittlicher Fehlerzahl $\leq 5 \%$ im Vigilanztest war mit TSESS 23,2\% bzw. TS-Young 23,5\% nicht signifikant different. Das Gleiche gilt für die Gruppe mit überdurchschnittlicher Fehlerzahl $>5 \%$. Die Kombination beider FB bei unterdurchschnittlicher Fehlerzahl ergab eine signifikante Zunahme von Patienten mit TS von $23,2 \%$ auf $31,4 \%$ aller Fälle $(p<0,001)$. Eine geringere, aber ebenfalls signifikante Zunahme der Fälle von $25 \%$ auf $28,1 \%$ ergab sich in der Gruppe mit überdurchschnittlicher Fehlerzahl im Vigilanztest.

In der Gruppe mit pathologischer Vigilanzstörung (Fehlerzahl $>9 \%$ ) lag die Anzahl der Patienten mit TS-ESS mit 50 (15,2\%) signifikant über derjenigen mit TS-Young mit 42 (12,8\%). Eine Kombination beider FB ergab gegenüber der ESS allein keinen weiteren prozentualen Zuwachs an TS ( $\bullet$ Tab.3).

\section{Diskussion}

$\nabla$

Häufig wird die Einschätzung der TS durch Patienten infolge verschiedener äußerer Faktoren und der Formulierung der Fragen in den Fragebögen beeinflusst. Die Antworten auf Fragen nach Wahrscheinlichkeiten des Einschlafens bei verschiedenen Situationen sind u.a. von dem Verstehen der Frage abhängig. Genaue- re Untersuchungen zur ESS bzgl. Verständnisschwierigkeiten bei jüngeren OSA-Patienten liegen nicht vor. Ältere Patienten konnten in $28 \%$ aller Fälle nicht alle Fragen der ESS beantworten [13]. Eine sehr viel konkretere Formulierung mit offensichtlicher „face validity“ wurde in der häufig zitierten epidemiologischen Studie von T. Young [9] verwendet. Hier werden gut fassbare und leicht zu beantwortende konkrete Fragen mit möglichen Ja-Nein Antworten gestellt.

Wir stellten bei dem Vergleich der beiden FB fest, dass sowohl mittels ESS als auch des 3 Fragen enthaltenden Fragebogens zur Schläfrigkeit bei knapp 50 Prozent aller Patienten mit OSA und einem AHI > 5 pathologische Schläfrigkeit gefunden wurde. Wir fragten uns weiter, ob durch eine Kombination der ESS mit dem Young-Fragebogen zusätzlich Patienten mit Verständnisschwierigkeiten bei der Beantwortung der ESS-Fragen erfasst werden. In dem Young-FB wird nicht wie in der ESS nach der Wahrscheinlichkeit, während des normalen Alltagsleben in der letzten Zeit einzuschlafen, sondern nach Häufigkeiten in einem definierten Zeitraum gefragt, sodass damit andere, differente Informationen geliefert werden.

Nachdem die ESS mit dem FB nach Young kombiniert wurde, konnte ein signifikant höherer Prozentsatz mit TS-Komb in der Patientengruppe mit einem $\mathrm{AHI}>5$ identifiziert werden. Es stellt sich deshalb die Frage, ob nicht zur Erhöhung der Sensitivität bei der Erfassung der subjektiven Schläfrigkeit zusätzlich der YoungFB zum Einsatz kommen sollte. Der zeitliche Aufwand für Arzt und Patient ist gering.

Wir fragten uns, ob die Patienten genauer charakterisiert werden können, deren Tagesschläfrigkeit nicht durch die TS-ESS, sondern erst durch die TS-Young-Kriterien erfasst werden.

Dazu bot sich der bei allen Patienten durchgeführte Vigilanztest zur Bildung von Untergruppen von OSA-Patienten an. Die Gruppeneinteilung erfolgte anhand des Referenz-Mittelwertes. Wir bildeten OSA-Gruppen mit geringerer und höherer Fehlerzahl im Vergleich zu diesem Mittelwert. In der Gruppe ohne Vigilanzstörung fanden wir durch die Kombination der FB im Vergleich mit der ESS allein den größten Zuwachs von 24,09\% auf 31,4\% der Patienten mit TS. Eventuell erfasst in dieser Gruppe die Frage nach unerfrischtem Aufwachen einen weiteren, weniger ausgeprägten Aspekt der Schläfrigkeit, der in der ESS nicht berücksichtigt wird.

Bemerkenswert ist, dass in der Gruppe mit eingeschränkter Vigilanz, d.h. mit einer Fehlerzahl $>9$ gegenüber TS-ESS, durch den zusätzlichen Young-FB keine weiteren Fälle mit TS hinzukommen.

Auffallend ist mit 21,3\% der geringe Prozentsatz aller OSA-Patienten mit eingeschränkter Vigilanz in dem von uns verwendeten CARDA-Vigilanztest. Wahrscheinlich ist ein einzelner Test

Tab. 3 Anzahl der Patienten mit unterdurchschnittlicher (<4,7\%), überdurchschnittlicher (>4,7\%) und signifikant erhöhter ( $>9 \%$ ) Fehlerzahl im Vigilanztest (CARDA) mit der ermittelten Zahl von Patienten mit pathologischer Tagesschläfrigkeit anhand der eingesetzten Fragebögen (ESS und Young), Prozentsatz in Klammern. Signifikante Differenz, wenn $\mathrm{p}<0,05$ (Chi²-Test).

\begin{tabular}{|c|c|c|c|c|c|c|}
\hline $\begin{array}{l}\text { Vigilanz-Test } \\
\text { Fehlende Reaktion } \\
\text { (\%) }\end{array}$ & $\begin{array}{l}\text { Patientenzahl } \\
\text { n (\%) }\end{array}$ & $\begin{array}{l}\text { TS-ESS } \\
n(\%)\end{array}$ & $\begin{array}{l}\text { TS-Young } \\
\text { n (\%) }\end{array}$ & $\begin{array}{l}\text { Chi²-Test } \\
\text { TS-ESS vs TS-Young } \\
\text { p }\end{array}$ & $\begin{array}{l}\text { TS-ESS und/oder } \\
\text { TS-Young vs TS-ESS } \\
\text { n (\%) }\end{array}$ & $\begin{array}{l}\text { Chi²-Test } \\
\text { TS-ESS vs Kombination } \\
\text { p }\end{array}$ \\
\hline$\leq 5$ & $192(58,5 \%)$ & $76(23,2 \%)$ & $77(23,5 \%)$ & 1,0 & $103(31,4 \%)$ & $<0,001$ \\
\hline$>5$ & $136(41,5 \%)$ & $82(25 \%)$ & $79(24,09 \%)$ & 0,68 & $92(28,05 \%)$ & 0,002 \\
\hline$>9$ & $70(21,3 \%)$ & $50(15,2 \%)$ & $42(12,8 \%)$ & 0,008 & $50(15,2 \%)$ & 1,000 \\
\hline
\end{tabular}


nicht ausreichend, um das gesamte Spektrum der Leistungseinschränkung zu erfassen. Erst durch den Einsatz mehrerer Tests zur selektiven und geteilten Aufmerksamkeit konnte gezeigt werden, dass 95\% der untersuchten OSA-Patienten Vigilanz- oder Aufmerksamkeitsdefizite aufwiesen [14].

Allerdings ist ein solcher Aufwand mit mehreren Tests in der Routine in Zeiten der Verknappung der personellen Ressourcen nicht realisierbar. Aufgrund der geringen Sensitivität eines einzelnen Vigilanztests bzgl. der Erfassung von Defiziten könnte mit geringem Aufwand ein zusätzlicher Fragebogen zur subjektiven Tagesschläfrigkeit (TS-Young) mit der ESS kombiniert werden. Dies würde vor allem bei fehlender Vigilanzstörung die Quote der Patienten mit Tagesschläfrigkeit erhöhen.

Ein weiterer Aspekt unseres Vergleichs des ESS-FB mit dem 3Item-FB nach Young ist die Tatsache, dass mit beiden Fragebögen der gleiche Prozentsatz von Patienten mit TS gefunden wurde. Selbst wenn die damalige epidemiologische Wisconsin-Studie mit dem heute am meisten benutzten ESS-FB durchgeführt worden wäre, würde dies an den Berechnungen zur Prävalenz von OSAS nichts ändern. Bei zusätzlicher Befragung mittels ESS hätte sich allerdings nach unseren Ergebnissen die Zahl der OSA-Patienten mit dem Symptom Schläfrigkeit um 11,9\% erhöht.

\section{Schlussfolgerungen}

Als einzelner Fragebogen zur Diagnostik der TS eignen sich sowohl die ESS als auch der Fragebogen nach Young mit gleicher Häufigkeit von Tagesschläfrigkeit. Kombiniert man die beiden Fragebögen, findet man in einem signifikant höheren Prozentsatz Hypersomnolenz, jeweils definiert anhand der angegebenen Grenzwerte. Vor allem bei Patienten mit geringer oder fehlender Vigilanzstörung findet man durch die Addition von 3 Fragen, die in der Wisconsin-Studie zur Frage der Prävalenz des OSAS verwandt wurden, einen im Vergleich zur ESS höheren Prozentsatz von OSA-Patienten mit Tagesschläfrigkeit.

\section{Interessenkonflikt}

$\nabla$

Die Autoren geben an, dass kein Interessenkonflikt besteht.

\section{Literatur}

1 Tregear S, Reston J, Schoelles $K$ et al. Obstructive sleep apnea and risk of motor vehicle crash: systematic review and meta-analysis. J Clin Sleep Med 2009; 5: 573-581

2 Johns MW. A new method for measuring daytime sleepiness: the Epworth Sleepiness Scale. Sleep 1991; 14: 540-545

3 Johns MW. Reliability and factor analysis of the Epworth Sleepiness Scale. Sleep 1992; 15: 376- 381

4 Johns MW. Daytime sleepiness, snoring, and obstructive sleep apnea. The Epworth Sleepiness Scale. Chest 1993; 103: 30-36

5 Johns MW. Sleepiness in different situations measured by the Epworth Sleepiness Scale. Sleep 1994; 17: 703 - 710

6 Rühle KH, Feier Ch, Galetke W et al. Analyse der 8 Fragen (Items) der Epworth-Sleepiness-Scale. Somnologie 2005; 9: 154-158

7 Johns M, Hocking B. Daytime sleepiness and sleep habits of Australian workers. Sleep 1997; 20: 844-849

8 Bloch KE, Schoch OD, Zhang JN et al. German version of the Epworth Sleepiness Scale. Respiration 1999; 66: $440-447$

9 Young T, Palta M, Dempsey J et al. The occurrence of sleep-disordered breathing among middle-aged adults. N Engl J Med 1993; 328: $1230-1235$

10 Adams N, Strauss M, Schluchter $M$ et al. Relation of measures of sleepdisordered breathing to neuropsychological functioning. Am J Respir Crit Care Med 2001; 163: 1626-1631

11 Randerath WJ, Gerdesmeyer C, Siller K et al. A test for the determination of sustained attention in patients with obstructive sleep apnea syndrome. Respiration 2000; 67: 526-532

12 Findley L, Unverzagt M, Guchu $R$ et al. Vigilance and automobile accidents in patients with sleep apnea or narcolepsy. Chest 1995; 108: 619-624

13 Frohnhofen H, Popp R, Willmann Vet al. Feasibility of the Epworth Sleepiness Scale in a sample of geriatric in-hospital patients. J Physiol Pharmacol 2009; 60: 0545-49

14 Mazza S, Pépin JL, Naëgelé B et al. Most obstructive sleep apnoea patients exhibit vigilance and attention deficits on an extended battery of tests. Eur Respir J 2005; 25: 75-80 